\title{
Dynamic Mission Control for UAV Swarm via Task Stimulus Approach
}

\author{
Haoyang Cheng ${ }^{*}$, John Page, John Olsen
}

School of Mechanical and Manufacturing Engineering, University of New South Wales, Sydney, 2052, Australia

\begin{abstract}
Interest in self-organized (SO), multi-robotic systems is increasing because of their flexibility, robustness, and scalability in performing complex tasks. This paper describes a decentralized task allocation model based on both task stimulus intensity and a responding threshold. The response threshold method was developed through observations of social insects. It allows a swarm of insects with a relatively low-level of intelligence to perform complex tasks. In this work, an agent based simulation environment is developed incorporating these ideas. The mission scenario simulated in this study is a wide area search and destroy mission in an initially unknown environment. The mission objectives are to effectively allocate a UAV swarm to both optimize coverage of the search space and attack a target. Rule based behaviours were used to create UAV formations. Two sets of simulations with different swarm size and target numbers were performed. The simulation results show that with task stimulus intensity and a responding threshold, the UAV swarm demonstrates emergent behaviour and individual vehicles respond adaptively to the changing environment.
\end{abstract}

Keywords UAV, Swarm Intelligence, Self-Organization, Simulation

\section{Introduction}

With recent technological advances in autonomous control and communication, multi-robotic systems are receiving a great deal of attention due to their increased ability to carry out complex tasks in a superior manner when compared to single-robotic systems. Multiple autonomous agents working in-group exceed the sum of the performance of the individuals. Currently, the human factor associated with the UAV (unmanned aerial vehicle) operators' workload is one of the key limitations to increasing future UAS (unmanned aerial system) effectiveness[1]. Such a requirement not only increases the expense of UAV operations, but also makes coordination among UAVs more complex. For these reasons, research is required to investigate methods of increasing UAV autonomy and interoperability, while reducing global communication and human operator reliance.

The cooperative control of UAVs is a complex problem that is dominated by uncertainty, limited information, and task coupling. Due to the complexities of the inherent problems, centralized decision and control algorithms are traditionally adapted to optimise timing and task constraints. One of the mission scenarios considered in the literature is cooperative moving target engagement. Kingston \&

* Corresponding author:

haoyang.cheng@student.unsw.edu.au (Haoyang Cheng)

Published online at http://journal.sapub.org/ajis

Copyright (C) 2012 Scientific \& Academic Publishing. All Rights Reserved
Schumacher in their paper solved this problem with a mixed integer linear program that addressed task timing constraints and agent dynamic constraints to generate a flyable path[2]. A genetic algorithm (GA) is used to efficiently search the space of possible solutions and provide the cooperative assignment[3]. Schumacher and Chandler also addressed the problem of task allocation for a wide area search munitions scenario[4]. A network flow optimization model is used to develop a linear program for optimal allocation of powered munitions to perform several tasks, such as search, classify, attack and finally damage assessment of potential targets. Cooperative task assignment within an adversarial environment was addressed in[5][6]. In their work, a cooperative task assignment was computed with the additional knowledge of the future implications of a UAV's actions. This was done to improve the expected performance of the other UAVs. In order to implement a centralized controller, consensus has to be reached under communication constraints. False information and communication delay strongly negates the benefits of cooperative control[7]. Alighanbari in his thesis[8] developed the unbiased distributed Kalman consensus algorithm which he proved converged to an unbiased estimate for both static and dynamic communication networks. Despite its benefits, the use of a centralized controller lacks significant robustness, is computationally complex and depends on high degree of global information. Such requirements make the implementation of centralized approach intractable in real-life missions.

In contrast, decentralized decision and control algorithms, 
trade optimality and predictability, with robustness and adaptation to environmental changes. A self-organized (SO) system, or swarm, is typically a decentralized control system made up of autonomous agents that are distributed in the environment and follow stimulus response behaviours[9]. Examples from social insects, such as foraging and the division of labour show that $\mathrm{SO}$ systems can generate useful emergent behaviours at the system level. Self-organised, swarm based systems do not require a centralised plan or deliberate action from individuals. They therefore have the potential to reduce mission planning and therefore the amount of intelligence required in control systems design. Moreover, self-organised, swarm based systems demonstrate robustness and scalability. This means that adding or subtracting agents to or from the system may not significantly affect the overall performance and emergent behaviours of the system.

Significant research effort has been invested in recent years into the design and simulation of intelligent swarm systems[10]. Intelligent swarm systems can generally be defined as decentralized systems, comprised of relatively simple agents which are equipped with the limited communicational, computational and sensing abilities required to accomplish a given task[11]. Gaudiano, et al. in their studies, tried to apply quantitative methodologies to evaluate the performance of UAV swarms under a variety of conditions[12]. In Price's research, ten self-organization rules were implemented whose weight factors were collected into a single fitness function. This function was further refined using a genetic algorithm within the simulation[13]. Another widely adopted mechanism is digital pheromone maps that imitate the foraging behaviour of ants. Digital pheromones are modelled on the pheromone fields of the individual vehicles. By synchronizing their maps the UAVs coordinate to avoid redundant searches[14]. Hauert, et al. had been investigating the potential of using a swarm of UAVs to establish a wireless communication network[15]. They applied artificial evolution to develop neuronal controllers for the swarm of homogenous agents.

This study designs a swarm controller to enable a swarm of UAVs to search for and attack targets. The concept of employing swarms of weapons was explored in[16]. In this concept, the individual of the swarm may be less capable than conventional weapons, but through cooperation across the swarm, the swarm exhibits behaviours and capabilities that can exceed those demonstrated by conventional systems that do not employ cooperation. To implement a swarm system, they designed a rule set of discrete behaviours, which was governed by interactive subsumptive logic. Building upon their research, we adopted an adaptive task controller based on task stimulus to optimize the performance of the swarm. In their design, once the targets were discovered, the weapons would home in on them. Through communication, other weapons had knowledge of how many weapons had been committed to a given target. Simple rules prevent excessive numbers of weapons from being expended on a single target. Our approach also takes into consideration the value of the targets and the time left in the mission. Thus the weapons would not be largely expended in the early stage of the mission, especially on low value targets, which leaves an inadequate number of UAVs to search for the remaining targets.

The remainder of this paper is structured as follows. Section II describes the mission in detail and the simulation tools used in this study. In section III, we present the task controller. The results of the simulation are given in section IV while section V concludes the paper.

\section{Simulation Environment}

In this section, we present the simulation tool and the overview of the simulated mission scenario.

\subsection{Simulation Tool}

Agent-based simulations of complex adaptive systems are becoming an increasing popular tool in the artificial life community. The application of agent-based simulations in combat modelling had been explored by Ilachinski[17]. He argued that agent-based models are most useful when they are applied to complex systems that can be neither wholly described nor can be built by conventional models based on differential equations. Agent-based models are designed to allow users to explore evolving patterns of system-level behaviour that derive collectively from low-level interactions among their agents.

In this study, the SWARM simulator, developed by the Santa $\mathrm{Fe}$ Institute was used to construct the simulation environment[18]. It provides a conceptual framework for designing, describing, and conducting experiments on agent-based models. This framework allows independent agents to interact via a schedule of discrete events. Bharathy et al. provided an architectural frame for generic agents, which comprised three external domains within an agent to interact with the agent's environment: perception domain, action domain and communication domain[19]. Within the SWARM framework, the agents communicate both with each other and with their environment via messages. SWARM supplies a basic system library that manages a dynamic list of objects and handles message passing between objects. In our design, two different objects were modelled, namely the UAVs and their targets. The internal functions of the objects, which describe the rule set of behaviours, handle the messages that are sent to the object and modify their internal states.

\subsection{Mission Description}

In applying these theoretical concepts to a UAV swarm application, we designed the following wide area search and attack scenario. This scenario aims to search for targets within the defined area and destroy or degrade targets. The following is a list of assumptions and functionalities we use in our simulation: 
- The vehicles considered in this study are relatively simple platforms with the smallest effective payload and with a minimal set of onboard detection devices to sense the environment and one another other. One concept of such platform is called the Low Cost Autonomous Attack System (LOCAAS), which has a range of 100 nautical miles as well as laser radar with automatic target recognition to identify potential targets[20].

- The mission area is defined as a rectangular region. Each UAV is aware of the terrain boundaries and will turn as it approaches a boundary to remain within the target area. The UAV has only enough fuel to fly over the mission area once.

- The UAV's were modelled as identical agents in the simulator. Each UAV is equipped with a global positioning system (GPS) navigation system for autonomous waypoint navigation, a circular sensor to detect the position of other UAVs within a specified radius and a limited range communication system.

- The UAV's fly at constant altitude but at a variable speeds. Their vehicle dynamics will be discussed in next section.

- The UAVs can change modes between search and attack. They can only change mode from search to attack once. The UAVs are themselves weapons which are destroyed in the attack process. The attack will have 80 percent chance of being successful.

- The targets are stationary and randomly distributed within target area. The potential targets could be relocatable air defence sites or vehicles in columns. The targets may be of high or low value. The stimulus of high value targets is set to be twice that of the low value targets. The onboard target recognition software has the ability to recognize the target type. The location and the nature of the targets are unknown to each UAV. Each target is assumed to be an array of ground vehicles or air defence units. In this simulation, each target array must be hit by 8 UAVs to be destroyed. The status of the target will degrade as the number of UAV attacks increase, so that the stimuli that attract UAV attacks will decrease. The mission control strategy based on task stimulus will be discussed in section III.

The mission was started with two groups of UAVs entering the mission area from the left side (Figure 1). The UAVs were assumed to be deployed from a cargo aircraft, though this could have been a ship or ground transport. The reason for deploying them in two groups was to encourage them distribute themselves evenly across the space during the mission. In[21], the feasibility of using cargo aircraft for the mass delivery of standoff munitions is investigated. The UAV's spread out immediately after release and form a loose formation using a six rule set (discussed in subsection B below) to hunt for targets (Figure 2). As shown in the figure, the targes within a circle were the ones that had been detected. The UAV's shown in green began the attack. Through communication, other UAVs that met certain proximity requirements became aware of the identified targets. However, the onboard task controller would decide whether to attack the targets or proceed to search for other targets.

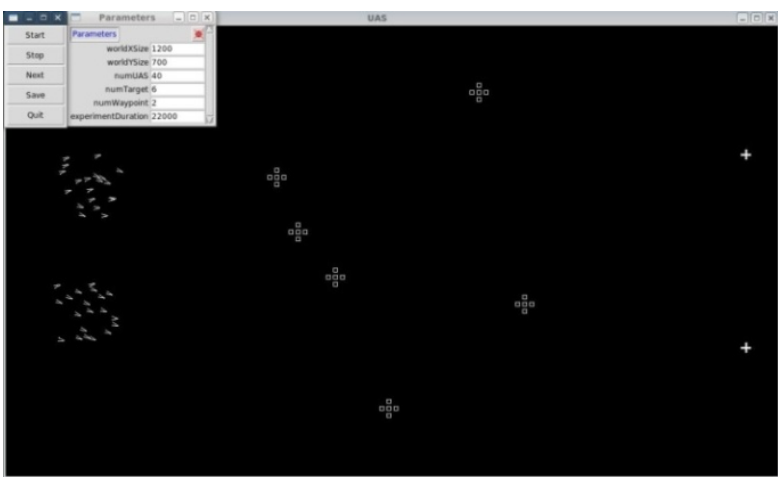

Figure 1. Mission Start

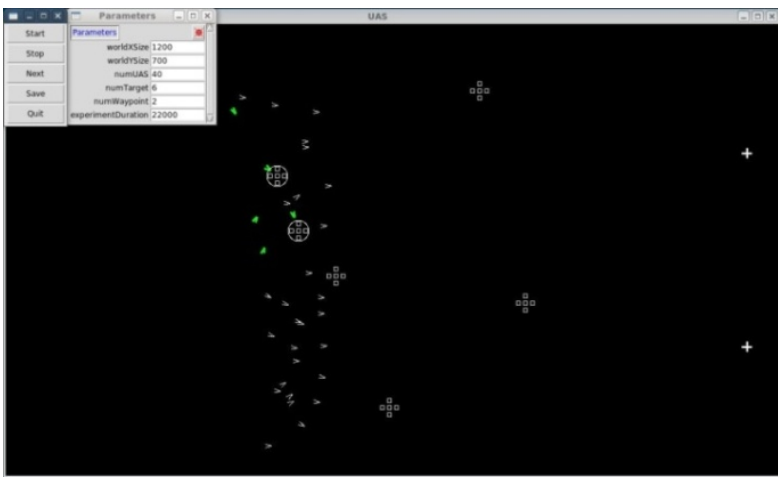

Figure 2. Search and Attack

\section{Dynamic Mission Control}

In this section, the rule set of behaviours and task controller is discussed.

\subsection{Rule sets of Movement}

The behaviours of the UAVs are built upon rule sets describing formation maintenance and target interaction. The mathematical definition of the rules is inspired from previous research[13]. This study extended the previous research by introducing two behavior logic modes on top of the rules, which are the search mode and target engagement mode. The switch between behavior modes is controlled by the task controller, which will be discussed in detail in next section. In this study, six rules are used to govern the movement of the vehicle: cohesion, alignment, separation, collision avoidance, target avoidance and goal seeking. When the UAVs are in the search mode, every rule is active, causing the vehicles to spread out while preserving intervehicle communication. Once the target engagement mode is triggered, the UAVs are committed to the attack phase and only collision avoidance and goal seeking rules are active.

Each of these rules is mathematically defined below.

1) Rule 1: Cohesion

The cohesion rule makes UAVs attract each other by orientating their acceleration vectors in the direction of the local flock center provided that the distance between them is greater than some set value. 


$$
\begin{gathered}
a_{1}^{i}=\operatorname{norm}\left(\frac{\sum_{j \in N B_{i}} P_{j}}{N_{i}}-P_{i}\right) \\
N B_{i}=\left\{U_{i}|| P_{U_{j}}-P_{U_{i}} \mid<r\right\},
\end{gathered}
$$

where $\left|P_{j}-P_{i}\right|$ means the distance between $U_{j}$ and $U_{i . .} N_{i}$ denotes the number of neighbours of UAV $i$.

2) Rule 2: Alignment

The alignment rule enables a UAV to align its heading and match its speed with its neighbours.

$$
a_{2}^{i}=\operatorname{norm}\left(\frac{\sum_{j \in N B_{i}} \frac{V_{i}}{P_{j}-P_{i}}}{N_{i}}\right) \text {, }
$$

1) Rule 3: Separation

The separation rule enables each UAV to maintain a distance between itself and its neighbours to prevent sensor overlap. It applies a repulsion vector that points away from neighbouring UAVs. The magnitude of the repulsion vectors are based upon a UAV's distance from its neighbors.

$$
\begin{gathered}
a_{3}^{i}=\operatorname{norm}\left(\sum_{j \in N B_{i}}\left(P_{j}-P_{i}\right) \cdot\left(1-\frac{P_{j}-P_{i}}{d_{s}}\right) \cdot f(x)\right) \\
f(x)=\left\{\begin{array}{l}
1,\left|P_{j}-P_{i}\right| \leq d_{s} \\
0,\left|P_{j}-P_{i}\right|>d_{s}
\end{array}\right\}
\end{gathered}
$$

2) Rule 4: Collision Avoidance

The collision avoidance rule works in a similar manner to separation rule but with a lower separation distance.

$$
\begin{gathered}
a_{4}^{i}=\operatorname{norm}\left(\sum_{j \in N B_{i}}\left(P_{j}-P_{i}\right) \cdot \frac{1}{\left(\frac{\left|P_{j}-P_{i}\right|}{d_{c}}\right)^{2}} \cdot f(x)\right) \\
f(x)=\left\{\begin{array}{l}
1,\left|P_{j}-P_{i}\right| \leq d_{c} \\
0,\left|P_{j}-P_{i}\right|>d_{c}
\end{array}\right\} .
\end{gathered}
$$

3) Rule 5: Target Avoidance

The target avoidance rule keeps a UAV from flying over detected targets. It works in a similar manner to the separation rule in that it provides a repulsion vector away from a known target.

$$
\begin{gathered}
a_{5}^{i}=\operatorname{norm}\left(\sum_{j \in N B_{i}}\left(P_{j}-P_{i}\right) \cdot\left(1-\frac{P_{j}-P_{i}}{d_{t}}\right) \cdot f(x)\right) \\
f(x)=\left\{\begin{array}{l}
1,\left|P_{j}-P_{i}\right| \leq d_{t} \\
0,\left|P_{j}-P_{i}\right|>d_{t}
\end{array}\right\} .
\end{gathered}
$$

4) Rule 6: Goal Seek

The goal seek rule guides a UAV to fly toward the next waypoint or target position if it is in attack mode.

$$
a_{6}^{i}=\operatorname{norm}\left(P_{w}-P_{i}\right) \text {. }
$$

The actions of the rules are weighted and summed to give a desired acceleration vector $\mathbf{a}$ for each UAV.

$$
a^{i}=\sum_{k=1}^{6} w f_{k} \cdot a_{k}^{i}
$$

The desired acceleration is limited by maximum linear and radial acceleration. The calculation of velocity and position use the following equations:

$$
\begin{gathered}
V(t+\Delta t)=V(t)+a \cdot \Delta t \\
P(t+\Delta t)=P(t)+V \cdot \Delta t .
\end{gathered}
$$

\subsection{Task Controller}

In Bonabeau, et al[10], a task allocation model based on a response threshold was developed through the observation of social insects. The response threshold refers to the likelihood of reacting to a task associated stimulus. A response threshold $\theta$, expressed in the units of stimulus intensity, is an internal variable that determines the tendency of an individual to respond to the intensity of the stimulus $S$ and perform the task. A response function $T_{\theta}(S)$ which is the probability of performing the task as a function of stimulus intensity $s$, is given by:

$$
T_{\theta}=\frac{S^{n}}{S^{n}+\theta^{n}},
$$

where $n>1$ determines the steepness of the threshold. As we can see from this function: for $S<<\theta$, the probability of engaging a task is close to 0 , and for $S>>\theta$, this probability is close to unity.

The mission objectives investigated in this paper focus on the effective allocation of weapons to maximise the coverage of the search space and attacks on targets. Use of flocking rules discussed above equally distributed the swarm in the search space and then re-adjusted its formation after an attack. One of the most challenging aspects that might affect the efficiency of the mission is how to allocate the swarm assets to each task. The tasks include search and the selection of the target to attack. An attack on a target causes the number of searching UAVs be reduced. An attack in the early stage of the mission will leave an inadequate number of UAV's to search and destroy the remaining targets. In order to use fewer vehicles to cover a greater target area, we designed a target area with a limited size. As a consequence, the UAVs fly over the search area once and so attacking targets after searching the whole area was not an option. It was important to make the UAVs decide when to attack and which target to attack. This study attempted to address these issues utilising the way self-organising and collective behaviour emerge from interactions among individuals and local environmental changes. We designed an adaptive task allocation model based on task stimulus intensity and responding threshold to maximise the performance of the swarm. 
This task control algorithm runs in each UAV at each time interval and calculates the probability that an individual should change its mode to attack. It is assumed that target array $T_{i}$ takes $N$ UAVs to attack successfully so as to be totally destroyed after it is detected. The discrete time dynamics of the stimulus intensity related to $T_{i}$ is updated by:

$$
S_{i}(t+1)=\left\{\begin{array}{c}
S(t)-\Delta N_{a c t}+\delta \cdot V, N_{a c t}<N \\
0, N_{a c t}=N
\end{array}\right\}
$$

where $N_{a c t}$ is the total number of UAVs that are assigned to attack target $T_{i}, \Delta N_{\text {act }}$ is the number of UAVs that attack $T_{i}$ and make a successful impact in the last time step, $\delta$ is the increase in stimulus intensity per unit of time and $V$ is the value of the target. If $\Delta N_{a c t}$ is equal to $N$, which means the target has already been totally destroyed, its stimulus becomes zero. The stimulus of high value targets will grow faster than low value targets once they are detected.

The probability $P_{i j}$ that the individual $U_{i}$ attacks a target $T_{j}$ is given by:

$$
P_{i j}=\frac{S_{j}^{2}}{S_{j}^{2}+\alpha \cdot \theta_{i j}^{2}}-\beta \cdot\left(1-e^{\left(\frac{-d_{i j}}{\gamma}\right)}\right),
$$

where $\alpha, \beta$ and $\gamma$ are coefficients that control the influences of $\theta \cdot d_{i j}$ denotes the distance between $U_{i}$ and $T_{j}$. The last term of equation (13) forces the UAVs to be more likely to attack the targets that are nearest to them if multiple targets are discovered. Each UAV at every time interval will decide whether to attack a target or proceed to search for other targets based on this probability. This controller will make a UAV choose a target with high stimulus intensity and spatially closer. Because the swarm self-organizes, the mission planning requirements are significant relaxed. Mission planning can be accomplished as an emergent behaviour, rather than as decisions sent to individual vehicles. The proposed task control algorithm is shown in figure 3 .

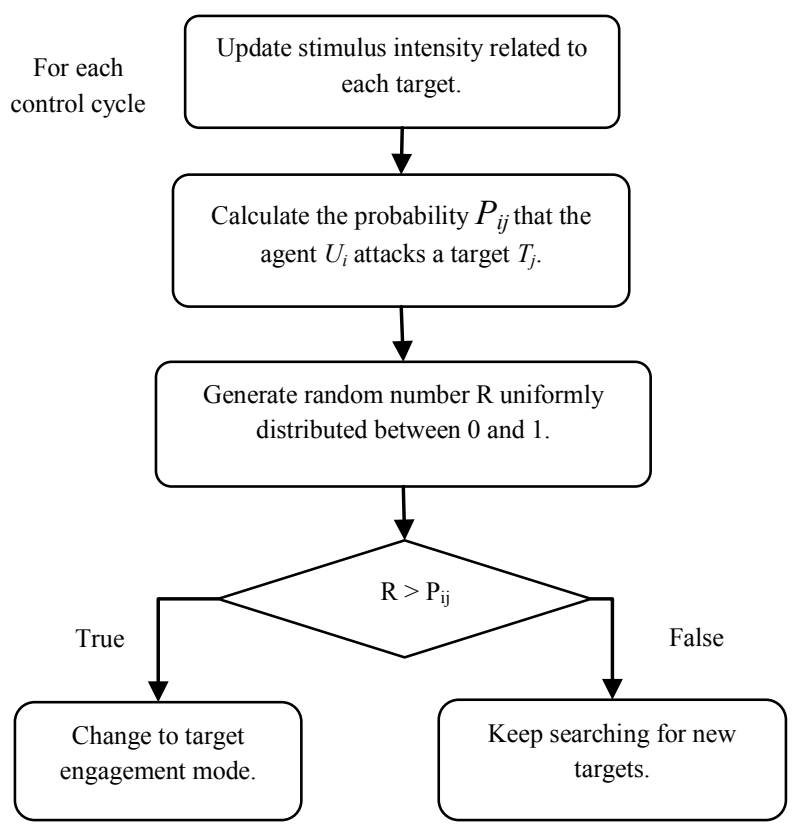

Figure 3. Task control flow chart

\section{Simulation Results}

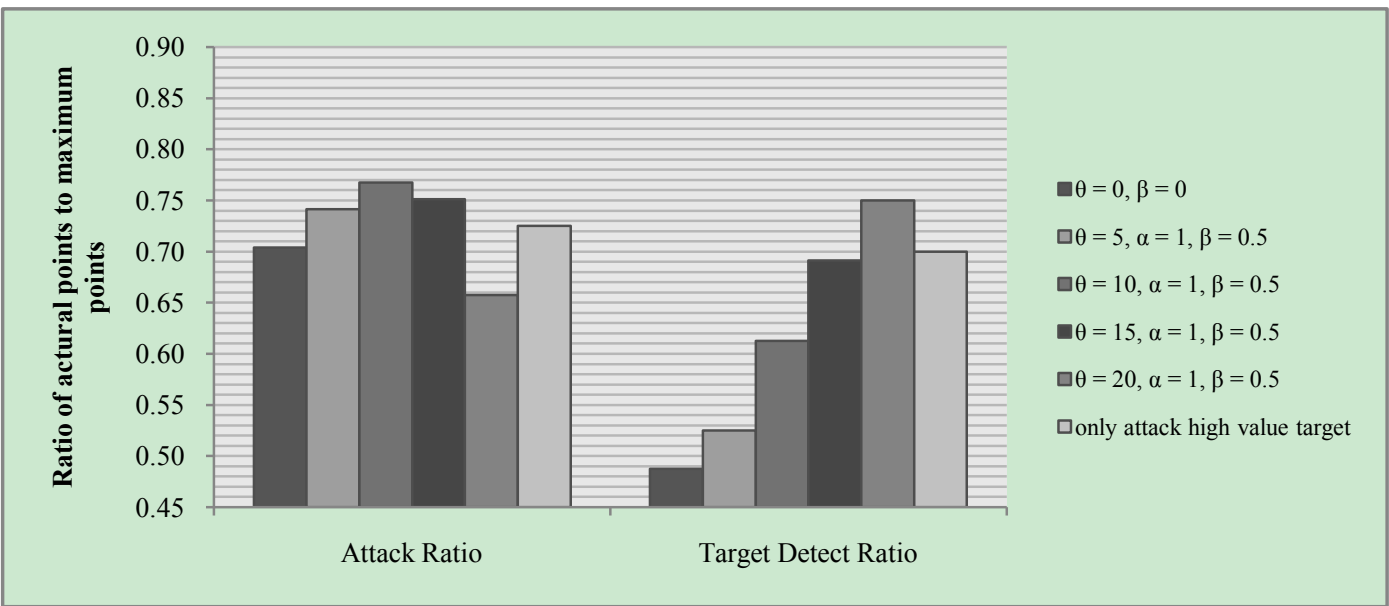

Figure 4. Simulation result with $40 \mathrm{UAV}$ s and 8 targets 


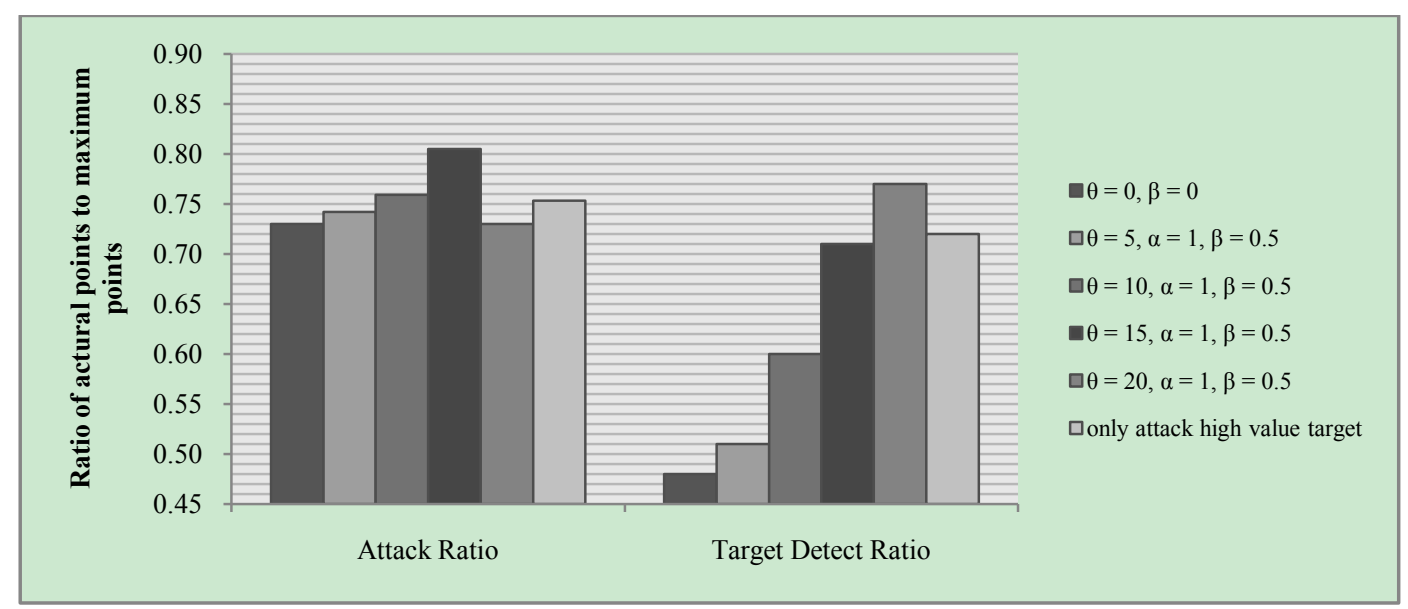

Figure 5. Simulation result with $50 \mathrm{UAVs}$ and 10 targets

Two sets of simulations with different swarm size and target numbers were performed to test the mission control algorithm, namely: (1) 40 UAVs and 8 targets and (2) 50 UAV's and 10 targets. The simulations were run in batch mode 30 times for each setting. The simulation results are shown in figures 4 and 5 . In the simulations, half of the targets were of high value while half were of low value. The position and status of the targets were unknown to the simulated UAVs before being detected. Each target was assumed to take potentially $8 \mathrm{UAVs}$ to be totally destroyed. Each attack had an 80 percent chance of being successful. To evaluate the mission performance, we assumed that an attack on a high value target received 2 hit points, while an attack on low value target received 1 hit point. The best situation was that all UAVs would attack a high value target and thus receive the most hit points. The attack ratio is actual points received in a mission divided by maximum points. The target detection ratio is the ratio of detected targets to total targets. The simulation results demonstrate the change of mission performance on attack and search as the value of $\theta$ in equation (13) is varied.

As is evident from the simulation results, both experiments with different swarm size and target numbers show the same pattern. We use $\theta=0, \beta=0$ as the baseline case. Because when $\theta=0, \beta=0$, the stimulus intensity of the target has no effect, so the swarm will attack every target immediately after it is detected until the target is totally destroyed. This is similar to the swarm behaviour used in previous research[13][16]. In this case, the mission performance of the swarm is not efficient because the UAVs attack any detected targets regardless of the value of the target and this may leave an inadequate number of UAVs to search for new target. As can be seen from the results, the target detection ration is extremely low. With $\theta$ increasing, the UAVs become less likely to attack a target with low stimulus intensity and pay more attention to high value targets. However, the target stimulus intensity will increase for each time step, the UAVs will still choose to attack low value target before running out of fuel if no more high value targets are detected. After $\theta$ passes a certain point, the attack ratio will fall because searching becomes more attractive than attacking. The last strategy we used for comparison was to let the UAVs only attack high value targets and ignore low value targets. As is shown, this strategy is not as efficient as the one with the optimum $\theta$ setting, because it runs the risk of wasting significant UAV resource if not enough high value targets are detected.

\section{Conclusions}

We have presented a formulation for a swarm of UAVs engaged in a wide area search and destroy mission in an unknown environment. We have used flocking behaviour to control the movement of the UAVs while searching the target area, as the rule based behaviour helps the vehicles to quickly disperse after deployment and readjust the formation as required. We have developed a decentralized mission control mechanism based on task stimulus intensity and a responding threshold, which can optimize the performance of the swarm. The simulation results show that, by using this mechanism, a swarm of UAVs demonstrates emergent behaviour through self-organisation, and individual vehicles respond adaptively to the changing environment.

The simulation scenario presented here is very simplified in comparison to a real mission. One weakness being the mission control mechanism only allocates UAVs to a certain target. A more sophisticated cooperative control algorithm is needed to control UAVs attacking multiple targets, especially when possible adversary action of the target is taken into consideration.

\section{Nomenclature}

$a=$ acceleration vector

$W_{i}=$ waypoint $i$

$N B_{i}=$ set of neighbours of UAV $i$

$P_{i}=$ position vector of agent $i$

$V_{i}=$ velocity vector of agent $i$

$r=$ interaction range between UAVs

$d_{c}=$ distance below which the collision avoidance rule take effect 
$d_{s}=$ separation distance from other UAVs

$d_{t}=$ separation distance from target

$\operatorname{norm}(V)=$ normalize vector $V$

$w f_{i}=$ weight factor for each rule

$S=$ stimulus intensity

$\theta=$ response threshold

$P_{i j}=$ probability that $U_{i}$ attack $T_{j}$

\section{REFERENCES}

[1] K. Heffner and F. Hassaine, "Towards Intelligent Operator Interfaces in Support of Autonomous UVS Operations," in 16th International Command and Control Research and Technology Symposium, Quebuc, Canada, 2011.

[2] D. B. Kingston and C. J. Schumacher, "Time-dependent cooperative assignment," in American Control Conference, 2005. Proceedings of the 2005, 2005, pp. 4084-4089 vol. 6 .

[3] T. Shima and C. Schumachery, "Assigning Cooperating UAVs to Simultaneous Tasks on Consecutive Targets using Genetic Algorithms," presented at the AIAA Guidance, Navigation, and Control Conference and Exhibit, San Francisco, California, 2005.

[4] C. Schumacher, et al., "Task allocation for wide area search munitions with variable path length," in American Control Conference, 2003. Proceedings of the 2003, 2003, pp. 3472-3477 vol.4.

[5] J. S. Bellingham, et al., "Cooperative path planning for multiple UAVs in dynamic and uncertain environments," in Decision and Control, 2002, Proceedings of the 41st IEEE Conference on, 2002, pp. 2816-2822 vol.3.

[6] M. Alighanbari and J. P. How, "Cooperative task assignment of unmanned aerial vehicles in adversarial environments," in American Control Conference, 2005. Proceedings of the 2005, 2005, pp. 4661-4666 vol. 7.

[7] T. Shima and S. Rasmussen, UAV Cooperative Decision and Control: Challenges and Practical Approaches: SIAM, Society for Industrail and Applied Mathemics, 2009.

[8] M. Alighanbari, "Robust and Decentralized Task Assignment Algorithms for UAVs," Doctor of Philosophy, Department of Aeronautics and Astronautics, Massachusetts Institute of Technology, 2007.
[9] S. Garnier, et al., "The biological principles of swarm intelligence," Swarm Intelligence, vol. 1, pp. 3-31, 2007.

[10] E. Bonabeau, et al., Swarm Intelligence- From Natural to Artificial Systems: Oxford University Press, 1999.

[11] Y. Altshuler, et al., "Swarm Intelligence-Searchers, Cleaners and Hunters," in Swarm Intelligent Systems, ed, 2006, pp. 93-132.

[12] P. Gaudiano, et al., "Control of UAV Swarms: What The Bugs Can Teach Us," presented at the 2nd AIAA "Unmanned Unlimited" Systems, Technologies, and Operations, San Diego, California, 2003.

[13] I. C. Price, "Evolving self-organized behavior for homogeneous and heterogeneous UAV or UCAV swarms," Master of Science, Department of Electrical and Computer Engineering, Air Force Institute of Technology, 2006.

[14] C. A. Erignac, "An Exhaustive Swarming Search Strategy based on Distributed Pheromone Maps," presented at the AIAA Infotech@Aerospace 2007 Conference and Exhibit, Rohnert Park, California, 2007.

[15] S. Hauert, et al., "Evolved swarming without positioning information: an application in aerial communication relay," Autonomous Robots, vol. 26, p. 11, 2009.

[16] D. R. Frelinger, et al., Proliferated Autonomous Weapons: An Example of Cooperative Behavior. Santa Monica, CA: RAND Corporation, 1998.

[17] A. Ilachinski, Artificial War: Multiagent-Based Simulation of Combat: \{World Scientific Publishing Company\}, 2004.

[18] (June 6). Swarm main page. Available: http://www.swarm. org/index.php/Swarm_main_page

[19] G. K. Bharathy, et al., "Agent Directed Simulation for Combat Modeling and Distributed Simulation," in Engineering Principles of Combat Modeling and Distributed Simulation, ed: John Wiley \& Sons, Inc., 2012, pp. 669-713.

[20] (June 6). Low Cost Autonomous Attack System (LOCAAS). Available: http://defense-update.com/products/1/locaas.htm

[21] M. E. Franke and G. Ari, "Use of Cargo Aircraft for Launching Precision-Guided Munitions," presented at the 42nd AIAA Aerospace Sciences Meeting and Exhibit, Reno, Nevada, 2004. 